\title{
VARIA
}

\section{MIGUEL PARRA Y NO RAMÓN BAYEU}

En 1958 y en estas mismas paginas de Archivo Español de Arte, se dieron a conocer unos pequeños lienzos, de colección particular barcelonesa, atribuyéndolos a Ramon Bayeu ${ }^{1}$.

El autor del breve artículo advertía que los cuatro lienzos que presentaba (Anunciación del Angel Zacarías, Nacimiento del Bautista, Predicación del Bautista y Degollación del Santo) eran de la misma mano y los consideraba, sin vacilar, obra del pintor aragonés, pues veía en ellos una gran similitud «en los escorzos, posición de manos, ojos y bocas de las figuras y en los paños que en grandes pliegues caen hacia el suelo», con los frescos del Pilar de Zaragoza (Regina Virginum, Regina Patriarcharum y Regina Confesorum), obra documentada del más joven de los cuñados de Goya.

De los cuatro lienzos, tres de formato vertical, casi cuadrado, eran sin duda compañeros, pues su carácter y dimensiones los ligaban muy directamente, mientras que el otro, -de formato absolutamente diverso, horizontal muy prolongado,-se separaba un tanto de sus presuntos compañeros, aunque parecía, desde luego, de la misma mano y al referirse también al Bautista, protagonista implícito o evidente de los otros tres, hacía pensar en que estuvieran de algún modo relacionados todos ellos La atribución fue recogida de inmediato por F. J. Sánchez Cantón ${ }^{2}$ que los calificó de «más enérgicos que delicados» y por J. L. Morales Marín ${ }^{3}$ que los incluyó en su Catálogo (n.․․ 21, 22, 23 y 24) con dimensiones claramente erróneas y fechándolos «hacia 1788».

Efectivamente, se trata de los bocetos preparatorios para una decoración mural homogénea, dedicada al santo precursor, pero no son obra de Ramón Bayeu, sino de otro artista más tardío en el tiempo, y aún escasamente conocido: el valenciano Miguel Parra (Valencia 1780-Madrid 1846).

\footnotetext{
${ }^{1}$ T. Larrucea Valdemoros, «Ramón Bayeu: Cuadros inéditos y cartones para tapices», A.E.A.,1958, p.313-319.

${ }^{2}$ F.J. Sánchez Cantón, Escultura y pintura del siglo XVIII. Francísco Goya, Ars Hispaniae, Vol. XVII. Madrid, 1965, p. 206, fig. 198 y 199.

${ }^{3}$ J. L. Morales y Marín, Los Bayeu, Zaragoza, 1979, p. 142-143.
} 


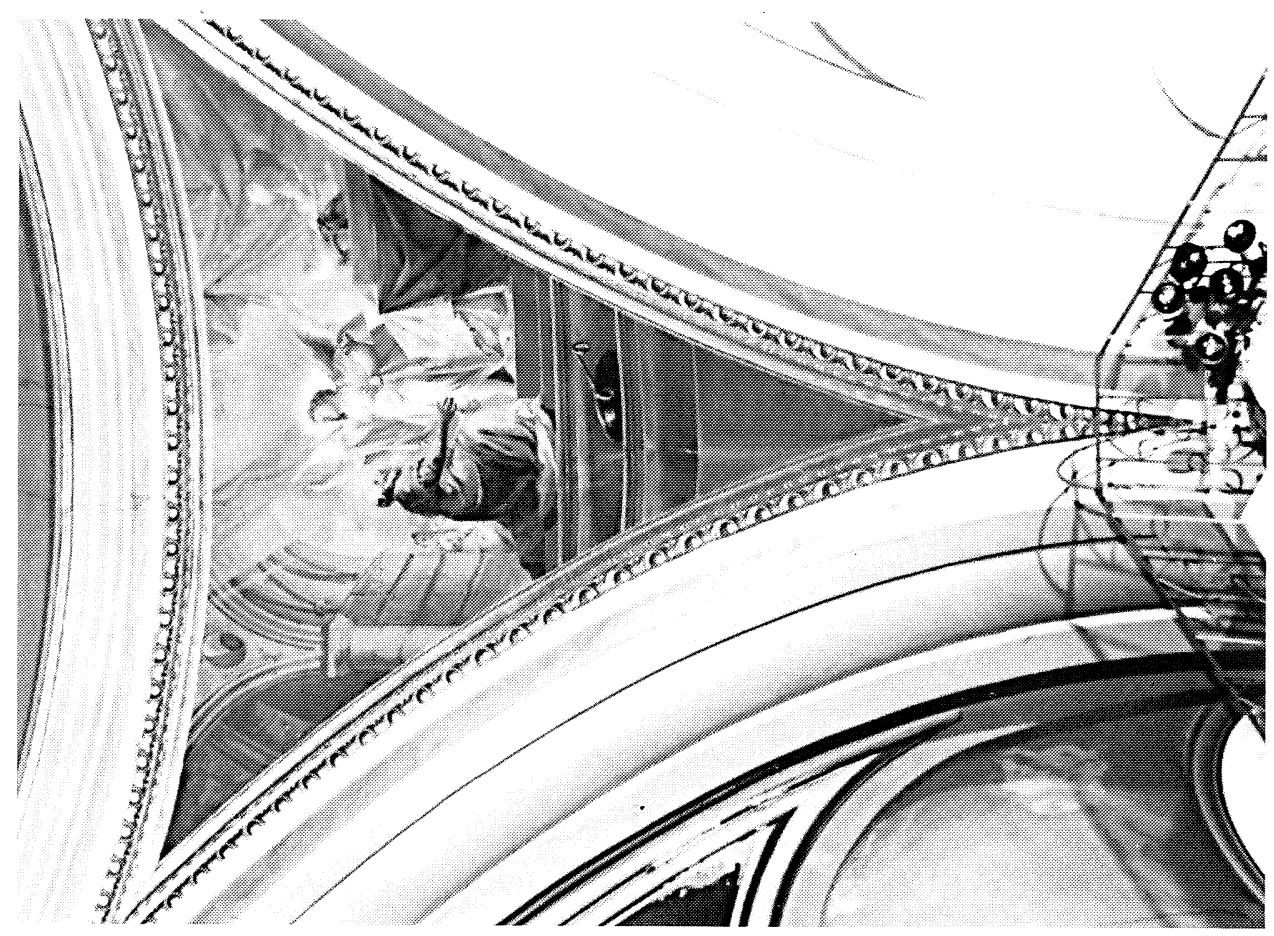

N

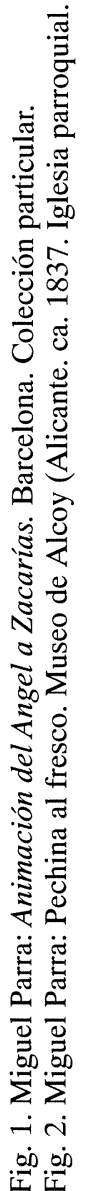



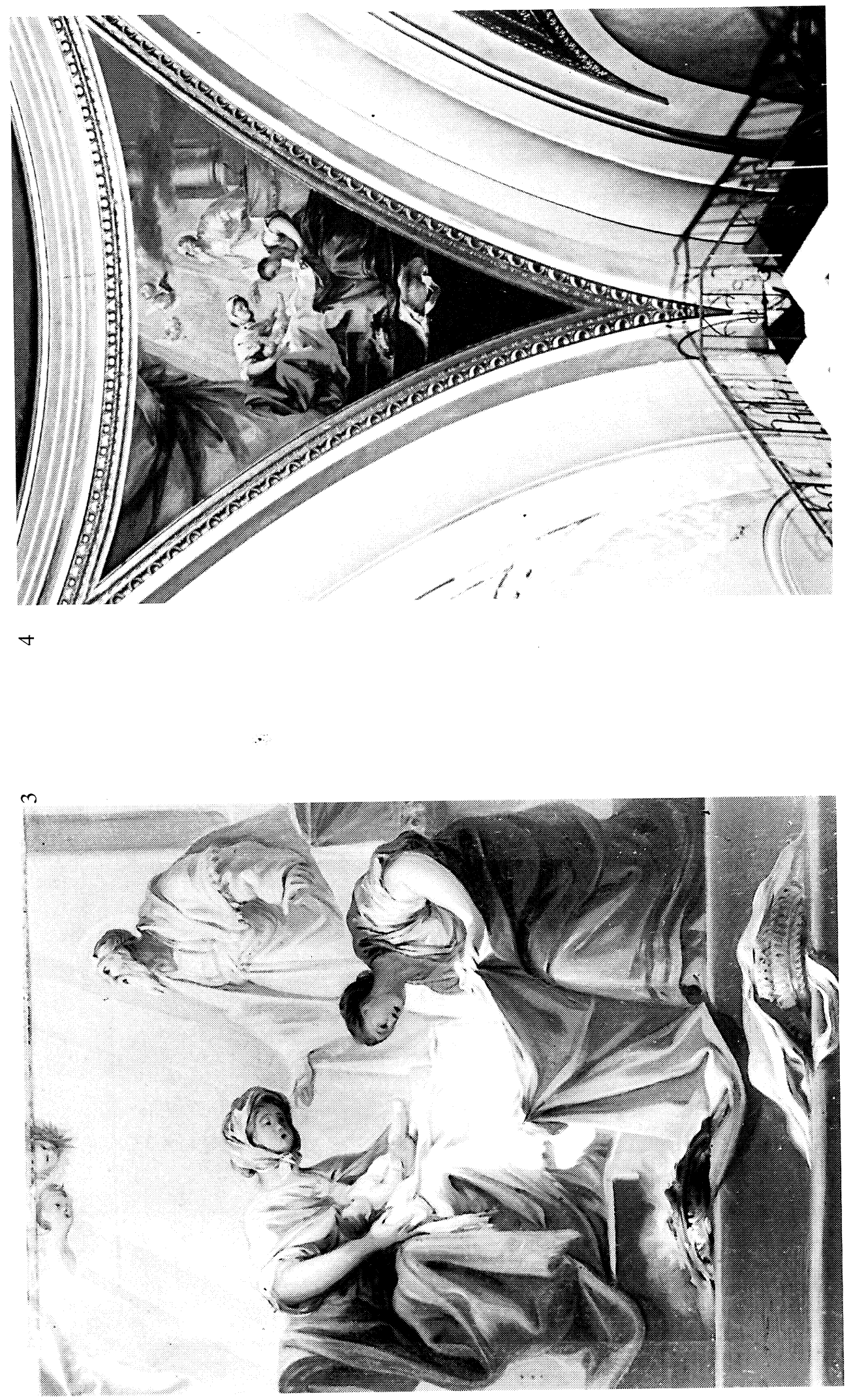

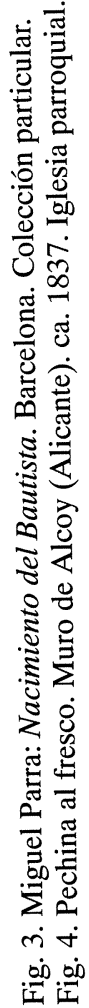


La reciente exposición Neoclásico y Academicismo en tierras alicantinas 1770-1850, celebrada en Alicante ${ }^{4}$ ha dado a conocer las pinturas al fresco de la iglesia parroquial de Muro de Alcoy, realizadas por Miguel Parra hacia 1837 -fecha en que la iglesia se concluyó y bendijo- que se corresponden fidelisimamente con lo que los bocetos apuntaban.

Las tres escenas de identico formato, se corresponden con tres de las pechinas, adaptando el formato rectangular del boceto a las proporciones triangulares de la pechina, con hábiles adiciones de fondo arquitectónico y escalera en el Anuncio del Angel Zacarías y en la Degollación de San Juan, y con vistosos cortinajes en el Nacimiento del Bautista. La cuarta pechina -para la que no se ha conservado el boceto- muestra el Bautismo de Cristo a la manera tradicional, inserto en un paisaje y con un fulgurante destello luminoso sobre la cabeza del Cristo.

La escena de la Predicación de San Juan Bautista en una de las bóvedas del crucero se corresponde también con absoluta fidelidad con el boceto. La figura del Santo, sentado en el centro, con el brazo derecho alzado y el gesto tronante, se destaca sobre fondo luminoso y los grupos de espectadores, a los lados, se disponen de modo que, efectivamente, evocan las ordenaciones de los Bayeu, y a lo lejos, los modos de Corrado Giaquinto.

La atribución de los frescos de la iglesia a Parra es bien antigua y no deja duda alguna respecto a su veracidad Ya Ossorio y Bernat, recogía en 1868, entre las obras del pintor La predicación de San Juan en la iglesia de Muro ${ }^{5}$ y luego se ha ampliado logicamente la paternidad a todo el conjunto ${ }^{6}$.

La atribución a Ramón Bayeu -nada convincente de todos modos- podría explicarse por el tono de barroquismo rezagado que presentan las composiciones, que no es, en modo alguno, único en la pintura valenciana del primer cuarto del siglo XIX.

Recuérdese que unos bocetos de Vicente Castelló para sus pinturas de la valenciana iglesia del Salvador fueron atribuidos a Francisco Bayeu ?

Parra, como Castelló, fue discípulo de Vicente López y a su través le llega mucho de la tradición del último barroco, atemperado por los rigores academicistas de Mengs, tal como lo vivieron los Bayeu y, sobre todo, Mariano Salvador Maella (1819) maestro, a su vez, de López.

La actividad de Parra como ordenador y catalogador del Museo Provincial de Valencia le hizo, también, sin duda, familiarizarse con las obras de un pasado ilustre y aunque se le suele considerar, sobre todo, como pintor de flores y como retratista ${ }^{8}$ estas obras que ahora se le devuelven ofrecen igualmente el testimonio de una viveza y de una habilidad compositivas nada desdeñables impregnadas, todavía, de gracia dieciochesca.

Alfonso E. PÉrez SÁnchez Universidad Complutense

\footnotetext{
${ }^{4}$ Producida por la Caja de Ahorros del Mediterráneo y el Instituto de Cultura Juan Gil Albert, de la Diputación de Alicante. Octubre 1997-Junio 1998.

${ }^{5}$ M. Ossorio y Bernat, Galería Biográfica de artistas españoles del síglo XIX. Madrid, 1868, 2a Edición, 1884 , p. 515.

${ }^{6}$ F. de Paula Momblanch y Gonzalbez, «El nuevo templo parroquial y la pena de excomunión. Los frescos de Miguel Parra» en Muro, Revista de Fiestas de Moros y Cristianos, 1977 y A. Espi Valdés, «Las artes plásticas alícantinas durante el síglo XIX» en Historia de la provincia de Alicante, Murcia, 1985, p. 408.

${ }^{7}$ A.E. Pérez Sánchez, «La personalidad artística de Vicente Castelló y Amat», Ars Longa, n. 1, Valencia, 1990. p. 19 y ss.

${ }^{8}$ Sobre Miguel Parra, véase además del clásico Ossorio y Bernat, ya citado, S. Aldana Fernández, Pintores valencianos de fores (1766-1866), Valencia, 1970, A.E. Pérez Sánchez, Natures mortes y flors del Museu de Bellas Artes de Valencia, Valencia, 1996, p. 105-111, y Sergio Pascual Garneria «Miguel Parra, Los retratos valencianos» Archivo de Arte Valenciano 1995. p. 77-85.
} 


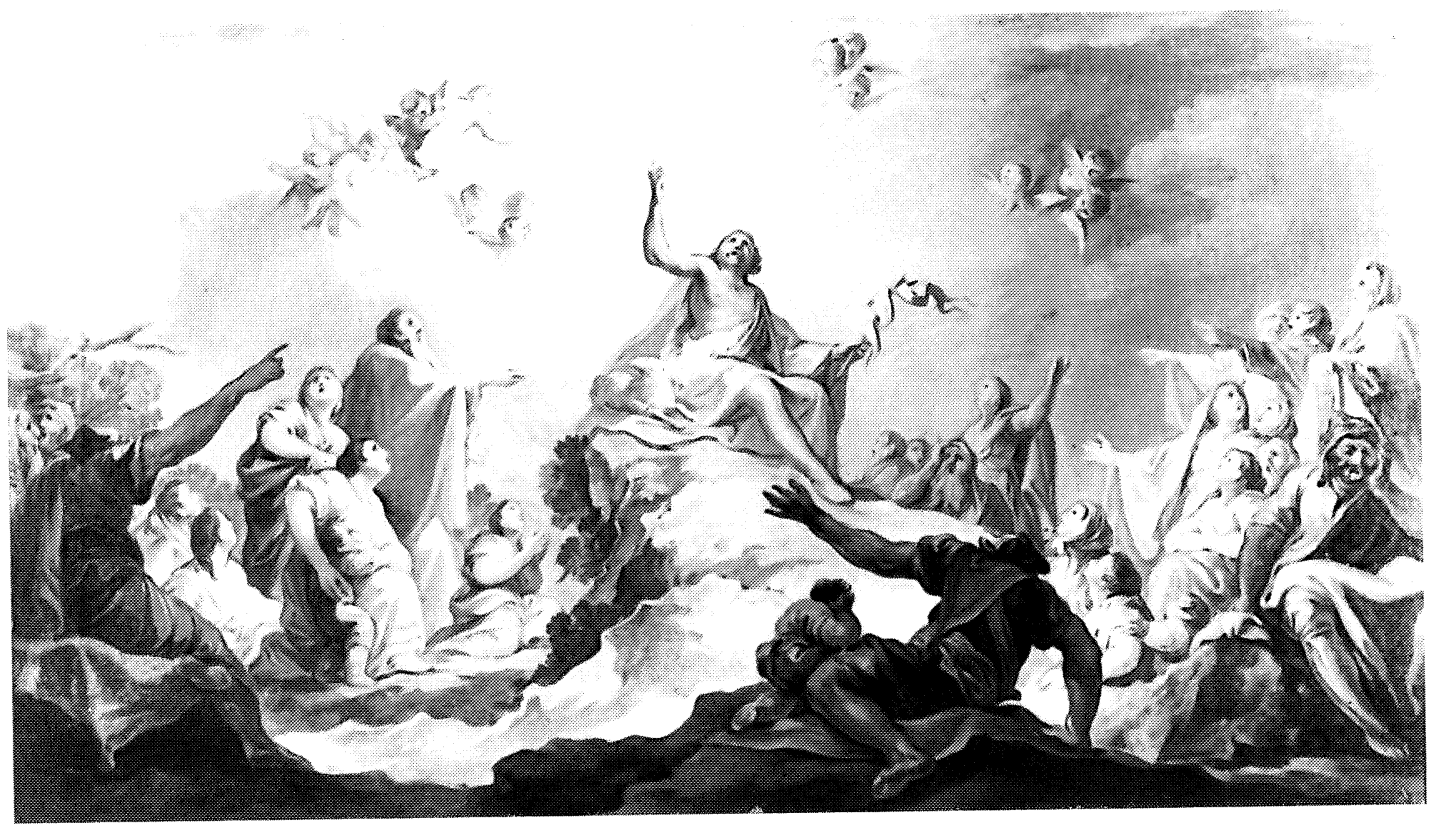

5

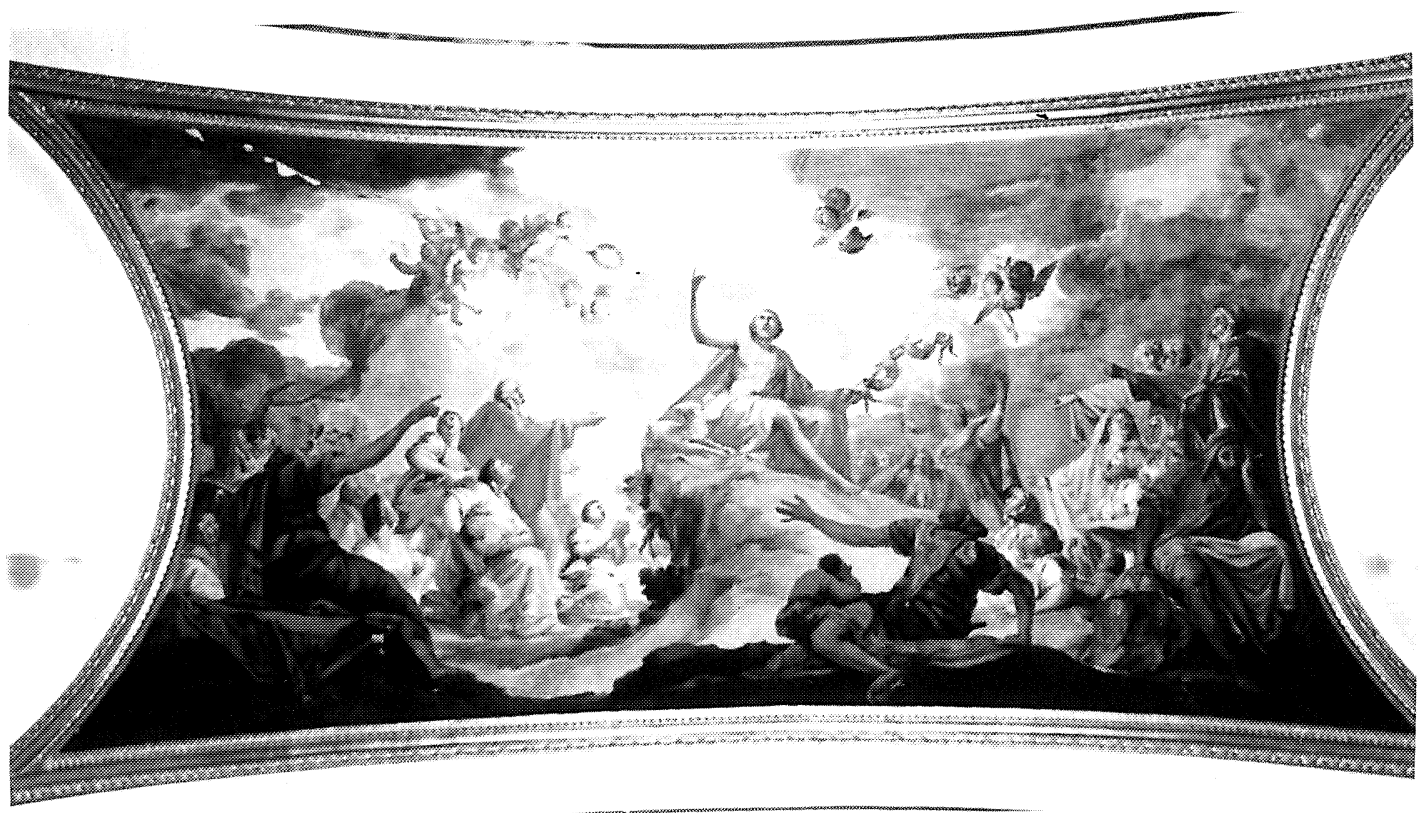

Fig. 5. Miguel Parra: Predicacción del Bautista. Barcelona. Colección particular. Fig. 6. Bóveda al fresco. Muro de Alcoy (Alicante). ca. 1837. Iglesia parroquial. 


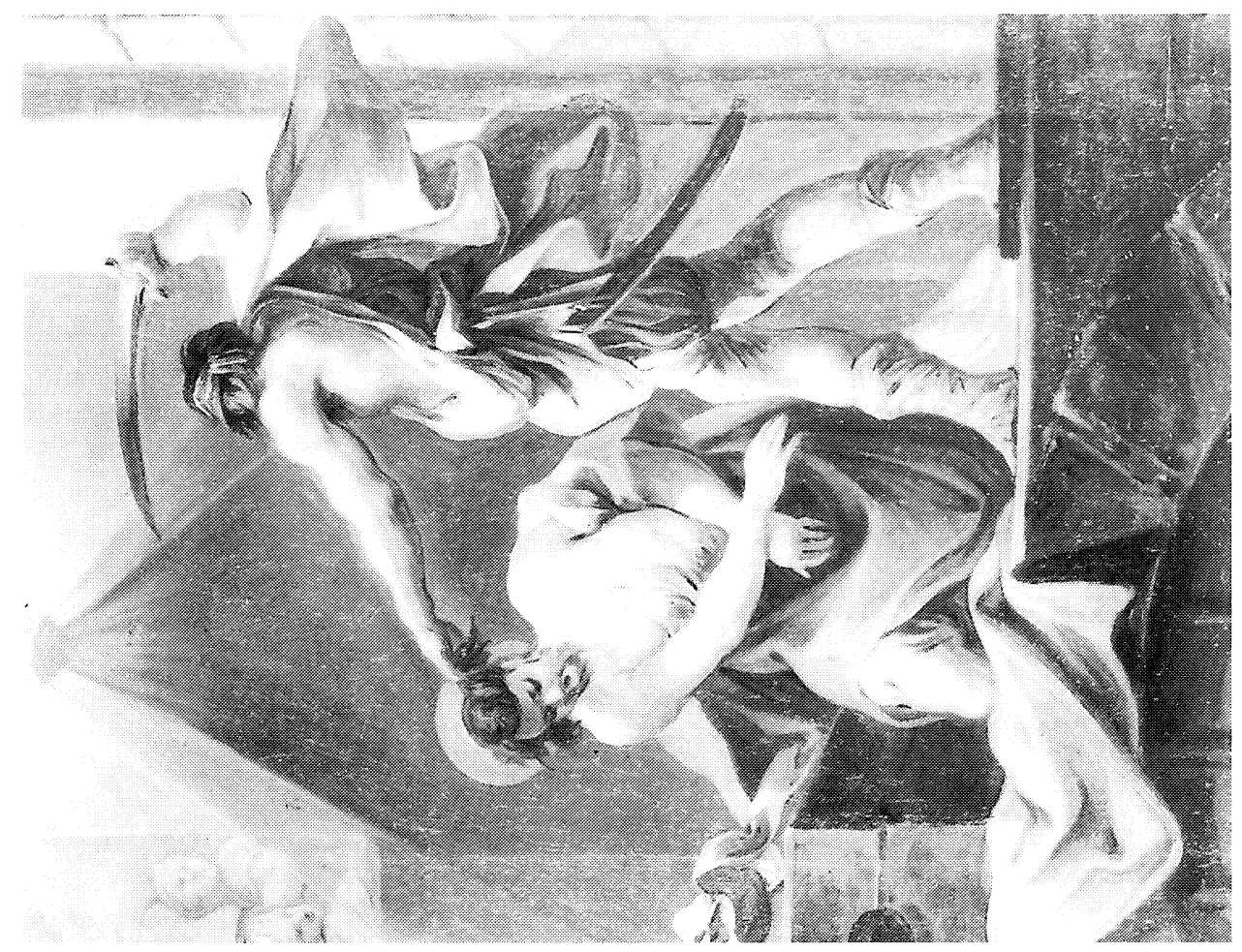

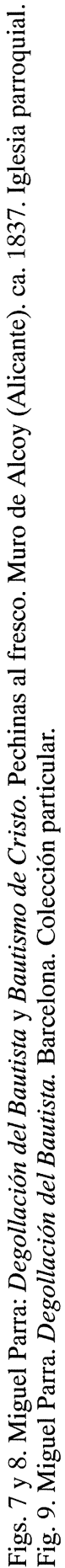

